\title{
The Significant Pathways and Genes Underlying the Colon Cancer Treatment by the Traditional Chinese Medicine PHY906
}

\author{
Ziyuan Su, ${ }^{1}$ Changyu Zhou, ${ }^{2}$ Shaoyou Qin, ${ }^{2}$ Erna Jia, ${ }^{2}$ and Zhenting Wu ${ }^{3}$ \\ ${ }^{1}$ Research Center of TCM, The Affiliated Hospital of Changchun University of Traditional Chinese Medicine, Changchun, China \\ ${ }^{2}$ Digest Department, China-Japan Union Hospital of Jilin University, Changchun, China \\ ${ }^{3}$ Digest Department, Heilongjiang Provincial Hospital, Heilongjiang, China
}

Correspondence should be addressed to Changyu Zhou; cyzhou@jlu.edu.cn

Received 21 February 2017; Accepted 6 April 2017; Published 15 May 2017

Academic Editor: Luciana Dini

Copyright (C) 2017 Ziyuan Su et al. This is an open access article distributed under the Creative Commons Attribution License, which permits unrestricted use, distribution, and reproduction in any medium, provided the original work is properly cited.

\begin{abstract}
Background. We attempted to explore the molecular mechanism underlying PHY906 intervention of colon cancer. Methods. The microarray data of tumors treated by PHY 906 and PBS alone were downloaded from the public Gene Expression Omnibus database. The dataset was further analyzed for the differentially expressed genes (DEGs) and their related biological functions were analyzed, followed by function and pathways. Protein-protein interaction (PPI) network was constructed and the significant nodes were screened by network centralities and then the significant modules analysis. Besides, they were clustered and transcriptional factors (TFs) were predicted. Results. The gene expression patterns changed induced by PHY906 treatment, including 414 upregulated and 337 downregulated DEGs. The biological process of response to steroid hormone stimulus and regulation of interferon-gamma production were significantly enriched by DEGs. Ezh2 (enhancer of zeste 2) was found to be the key node in PPI network. There are 12 significant TFs predicted for module 1 genes and 3 TFs for module 2 genes. Conclusions. PHY 906 treatment may function in protecting the epithelial barrier against tumor cell invasion by modulating IFN- $\gamma$ level and mediating cancer cell death by activating the response to steroid hormone stimulus and activating the response to steroid hormone stimulus. E2f1, Hsfy2, and Nfyb may be therapeutic targets for colon cancer. PHY906 showed treatment efficacy in modulating cell apoptosis by intervening interferongamma production and response to steroid hormone stimulus. Ezh2 and its TFs such as E2f1, Hsfy2, and Nfyb may be the potential therapeutic targets for anticancer agents development.
\end{abstract}

\section{Background}

Colon cancer is the common tumor derived from gastrointestinal systems, which has affected 1.4 million new cases in 2012 and results in 694,000 deaths globally [1]. It has been proposed to be the third most common cancer of all cases and the second most common cause of cancer related deaths [2]. The standard treatment used for colon cancer is the combination of surgery, chemotherapy, or radiation therapy [3]. The survival rate for cases after treatment is poor, which may be attributed to the treatment-related toxicity. The traditional Chinese medicine (TCM) as the adjuvant treatment plays a critical role in decreasing the complications of chemotherapy and radiation therapy in colon cancer patients [3].
PHY906 is a TCM prepared from four medical herbs, such as Glycyrrhiza uralensis Fisch. (G), Paeonia lactiflora Pall. (P), Scutellaria baicalensis Georgi (S), and Ziziphus jujuba Mill. (Z). It has been widely used for treating gastrointestinal symptoms in Eastern countries for about 1800 years [4]. In recent years, the four-herb TCM has been suggested to be used for cancer therapy for its potent anticancer properties [5]. It plays a modulator role in gastrointestinal toxicity induced by the chemotherapeutic drug irinotecan (CPT-11) of patients with colorectal cancer [6]. However, the mechanism underlying the antitumor modulation of PHY906 in chemotherapy has not been suggested clearly.

Currently, microarray technology has been widely applied to elucidate the tumor progression in different conditions. Wang et al. developed the microarray data of mice 
bearing colon tumor following treatment with PHY906 plus CPT-11 and suggested that the combination of PHY906 and CPT-11 created a unique response in colon tumors [7].

In order to explore the potential mechanism of PHY906 in treating colon tumors, we reanalyzed the published microarray data of colon tumor treated with PHY906 and PBS (Phosphate Buffered Saline) individually. The differentially expressed genes (DEGs) induced by PHY906 intervention were identified and then the DEGs related function and pathways were analyzed.

\section{Materials and Methods}

2.1. Microarray Data Acquisition. In order to evaluate the effect of PHY906 on colon cancer, we mined the gene expression data from fully public GEO database. The microarray data with the series number GSE25192 were downloaded from GEO database, which was contributed by Wang et al. [7]. The microarray data included 32 colon tumor samples from tumor-bearing mice treated with Phosphate Buffered Saline (PBS) $(n=10)$, PHY-906 $(n=9)$, CPT-1 $(n=10)$, and the combination of PHY-906 and CPT-1 $(n=9)$. In order to explore the therapeutic effect of PHY-906, we only selected the dataset of 9 samples of BDF-1 mice bearing colon 38 tumors treated with PHY906 and 10 tumor samples of those treated with PBS as controls for further analysis. The raw data were downloaded based on the platform of CCDTM Mm-CCDTM36k (derived from GPL5960).

2.2. Data Preprocessing and DEG Analysis. The raw file data (in gpr format) were preprocessed in limma package [8], including background correction, expression normalization, and microarray data condensation. Then, the DEGs in PHY906 treated samples compared with PBS samples were analyzed. $P$ values for DEGs were calculated with nonpaired $t$-test with the application of limma package. For DEGs identification, the cutoff value was set at $P<0.05$ and $\mid \log _{2} \mathrm{FC}($ fold change $) \mid \geq 0.58$.

2.3. Function Enrichment Analysis. The gene sets that share the common biological function and pathways can be analyzed by DAVID online tool [9]. In the present study, the up- and downregulated DEGs were subjected to function enrichment analysis, respectively. The count $\geq 2$ and $P$ value $<0.05$ were set as the cutoff value.

2.4. Protein-Protein Interaction Network and Significant Nodes Analysis. The protein-protein interactions (PPIs) were predicted based on the information deposited in STRING (Search Tool for the Retrieval of Interacting Genes/Proteins) database [10]. The protein pairs collection was based on the evidence of neighborhood, gene fusion, cooccurrence, coexpression experiments, databases, and text mining. The gene sets with differential expression were submitted to STRING online tool. The species was set as Mus and PPI score (medium confidence) as 0.4 . The PPI network comprised of DEG nodes was visualized by Cytoscape software.
The essential nodes within PPI network were analyzed based on 3 network centralities, such as degree centrality [11], betweenness centrality [12], and subgraph centrality [13]. The multiple centrality measures were conducted by CytoNCA plugin [14]. Degree and subgraph were calculated to evaluate the significance of network nodes. In addition, the betweenness was obtained to measure the influence of a node on the spread of information throughout the network.

2.5. Module Analysis. The gene sets in the modules of network generally share the common biological process. The significant modules in PPI network were analyzed by hierarchical cluster analysis with the application of ClusterONE [15] and the KEGG (Kyoto Encyclopedia of Genes and Genomes) pathways for module genes were annotated. The modules with $P<2.0 E-6$ were considered as significant.

2.6. Prediction of Transcription Factors for Module Genes. The TFs that played a regulatory role in module were predicted by iRegulon Cytoscape plugin [16]. The TF-target gene interactions were collected from Transfac, Jaspar, Encode, Swissregulon, and Homer database. The significant TFs were analyzed based on the TF binding motif enrichment analysis. The parameters were set as follows: minimum identity between orthologous genes: 0.05 and maximum false discovery rate on motif similarity: 0.001. Finally, the Normalized Enrichment Score (NES) was output. TF-target gene pairs with NES $>5$ were selected and visualized in PPI network modules.

\section{Results}

3.1. DEGs Identification. Total 751 DEGs were identified in PHY906 treated colon tumor tissues, including 414 up- and 337 downregulated DEGs. As shown in Figure 1, volcano plot illustrates that the expression patterns of genes are altered in tumors after PHY906 treatment compared with PBS treatment.

3.2. Function Enrichment Results. The DEGs were enriched in 3 GO Categories, such as biological process (BP), cellar component (CC), and molecular function (MF) (Table 1). The upregulated DEGs were closely related to response to steroid hormone stimulus related BP, proteinaceous extracellular matrix related CC, and di- and trivalent inorganic cation transmembrane transporter activity related MF. The downregulated genes were significantly enriched in regulation of interferon-gamma production (BP), cell fraction (CC), and cation:amino acid symporter activity (MF). The significant pathway for downregulated DEGs was tryptophan metabolism, while there was no pathway enriched by upregulated DEGs.

3.3. PPI Network and Significant Nodes. Based on the protein interactions corresponding to DEGs, the PPI network was constructed, which was comprised of 365 DEGs and 1005 protein pairs (Figure 2). Then, the key nodes in PPI network were analyzed by degree, betweenness, and subgraph centrality. As shown in Table 2, Acly (ATP citrate lyase), Rhoa (ras homolog 
TABLE 1: The overrepresented GO terms and pathways for differentially expressed genes.

\begin{tabular}{|c|c|c|c|c|}
\hline & Category & Term & Count & $P$ value \\
\hline \multirow{9}{*}{ Up } & \multirow{3}{*}{$\mathrm{BP}$} & GO:0048545 response to steroid hormone stimulus & 6 & $4.38 E-03$ \\
\hline & & GO:0007131 reciprocal meiotic recombination & 3 & $1.06 E-02$ \\
\hline & & GO:0000279 M phase & 11 & $3.21 E-02$ \\
\hline & \multirow{3}{*}{ CC } & GO:0015629 actin cytoskeleton & 10 & $1.01 E-02$ \\
\hline & & GO:0005578 proteinaceous extracellular matrix & 12 & $1.56 E-02$ \\
\hline & & GO:0044449 contractile fiber part & 6 & $1.74 E-02$ \\
\hline & \multirow{3}{*}{ MF } & GO:0015082 di- and trivalent inorganic cation transmembrane transporter activity & 5 & $2.22 E-03$ \\
\hline & & GO:0017076 purine nucleotide binding & 46 & $1.47 E-02$ \\
\hline & & GO:0030674 protein binding, bridging & 4 & $1.95 E-02$ \\
\hline \multirow{10}{*}{ Down } & \multirow{3}{*}{$\mathrm{BP}$} & GO:0032649 regulation of interferon-gamma production & 4 & $6.74 E-03$ \\
\hline & & GO:0019439 aromatic compound catabolic process & 3 & $1.39 E-02$ \\
\hline & & GO:0048535 lymph node development & 3 & $2.19 E-02$ \\
\hline & \multirow{3}{*}{ CC } & GO:0000267 cell fraction & 18 & $1.62 E-03$ \\
\hline & & GO:0005624 membrane fraction & 16 & $2.28 E-03$ \\
\hline & & GO:0005626 insoluble fraction & 16 & $3.17 E-03$ \\
\hline & \multirow{3}{*}{ MF } & GO:0005416 cation:amino acid symporter activity & 3 & $6.38 E-03$ \\
\hline & & GO:0005506 iron ion binding & 11 & $1.02 E-02$ \\
\hline & & GO:0015171 amino acid transmembrane transporter activity & 4 & $2.37 E-02$ \\
\hline & Pathway & mmu00380:tryptophan metabolism & 5 & $3.10 E-03$ \\
\hline
\end{tabular}

Up means upregulated genes, and down represents downregulated genes.

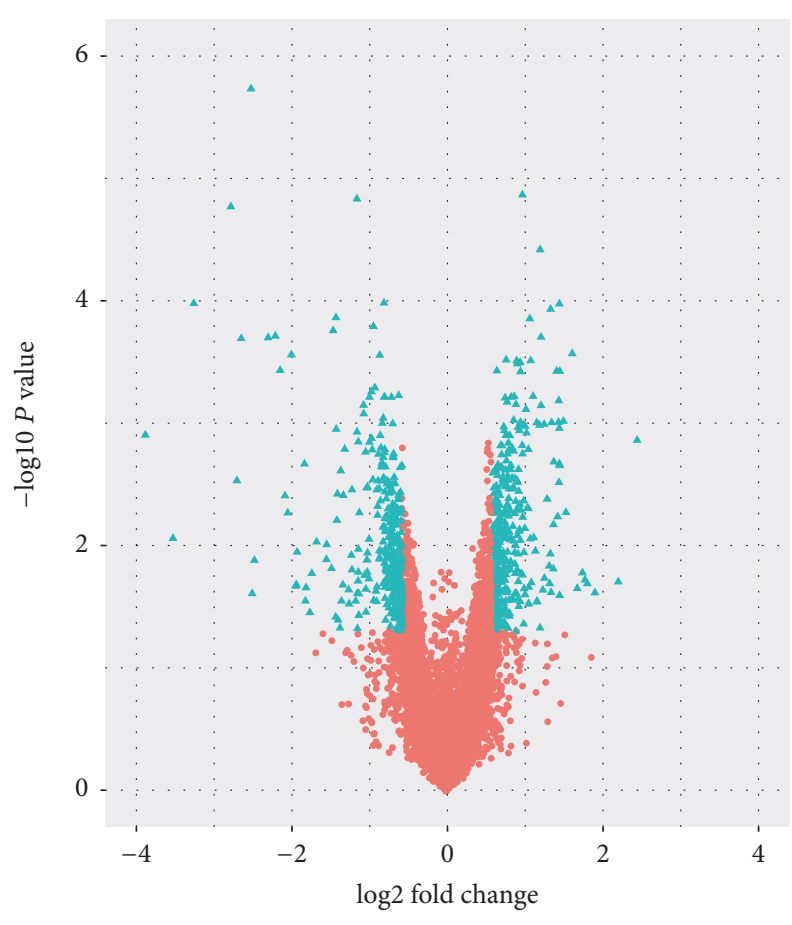

Threshold

- False

$\triangle$ True

FIGURE 1: Volcano plot of the differential expression of genes.

family member A), Erbb3 (erb-b2 receptor tyrosine kinase 3), Ezh2 (enhancer of zeste 2), and Rps27a (ribosomal protein
TABLE 2: Top 10 significant nodes based on three network centralities.

\begin{tabular}{lccccc}
\hline Nodes & Subgraph & Nodes & Degree & Nodes & Betweenness \\
\hline Olfr20 & 9011718 & Acly & 36 & Acly & 28651.502 \\
Olfr1022 & 9011523 & Rhoa & 25 & Rhoa & 22965.008 \\
Olfr281 & 8964057 & Erbb3 & 25 & Rps27a & 13976.256 \\
Olfr26 & 8964057 & Rad51 & 23 & Trip13 & 12352.914 \\
Olfr1100 & 8964057 & Ezh2 & 22 & Tex11 & 11707.876 \\
Olfr1463 & 8964057 & Ccnb2 & 21 & Erbb3 & 10906.968 \\
Olfr1054 & 8964057 & Rps27a & 21 & Olfr20 & 10419.8125 \\
Olfr1032 & 8964057 & Olfr20 & 20 & Anln & 8507.561 \\
Olfr16 & 8964057 & Olfr1022 & 20 & Mmp2 & 8288.151 \\
Olfr301 & 8964057 & Rrm1 & 20 & Ezh2 & 7548.0337 \\
\hline
\end{tabular}

S27a) are the common key nodes according to both degree and betweenness. Olfr20 (olfactory receptor 20) and Olfr1022 (olfactory receptor 1022) are the significant nodes with high scores based on subgraph and degree.

3.4. Modules and TFs. After cluster analysis, 2 significant modules were screened. Function enrichment analysis showed that the module 1 genes were closely related to sensory perception of smell, sensory perception of chemical stimulus related biological process, olfactory receptor activity related $\mathrm{MF}$, and olfactory transduction pathway. The module 2 genes were significantly enriched in cell cycle and DNA replication related $\mathrm{BP}$ and pathways (Table 3 ).

Figure 3 illustrates that there are 12 significant TFs and 62 TF-target gene pairs in module 1 . In module 2, 3 TFs are 


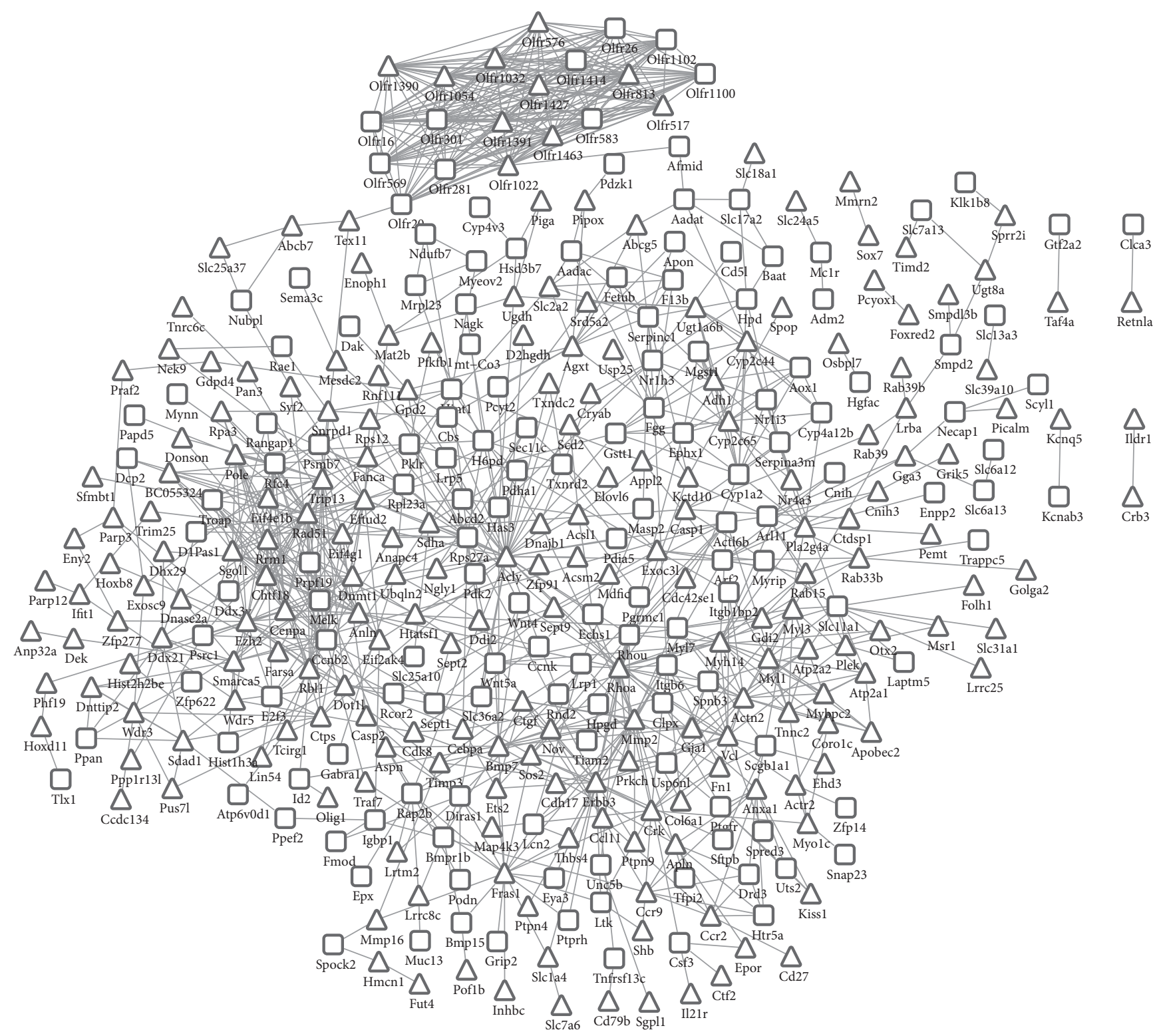

FIGURE 2: The protein-protein interaction network for differentially expressed genes. The trigon represents upregulated genes and the square represents downregulated genes.

predicted for module genes and involved in 34 TF-target pairs (Figure 3).

\section{Discussion}

PHY906, as one of the traditional Chinese herbal formulations, possesses the advantage in decreasing the side effect and complications following chemo- and radiotherapies [17]. PHY906 has been commonly used as the adjuvant agent for treatment of various cancers, especially for colon cancer. The understanding of the role of PHY906 in colon cancer treatment is insufficient from current knowledge. In order to provide novel insight to understand the molecular mechanism of PHY906 in treating colon cancer, the microarray data from the colon tumors treated with PHY906 alone were further analyzed.

Our data demonstrated that the PHY906 administration induced significant alterations in gene expression levels of colon tumors comparable to PBS treatment. In the presents study, total 751 DEGs were screened out in PHY906 treated tumors including 414 upregulated DEGs and 337 downregulated ones. Function analysis showed the regulation of interferon-gamma production and response to steroid hormone stimulus were dysregulated in PHY906 treated tumors.

The biological process of regulation of interferongamma (IFN- $\gamma$ ) production is significantly enriched by downregulated genes. It has been reported that IFN- $\gamma$ affects 
TABLE 3: The significant GO terms and pathways for genes in modules.

\begin{tabular}{|c|c|c|c|c|}
\hline & Category & Term & Count & $P$ value \\
\hline \multirow{7}{*}{ Module 1} & \multirow{3}{*}{$\mathrm{BP}$} & GO:0007608 sensory perception of smell & 20 & $2.10 E-21$ \\
\hline & & GO:0007606 sensory perception of chemical stimulus & 20 & $7.28 E-21$ \\
\hline & & GO:0007600 sensory perception & 20 & $1.62 E-19$ \\
\hline & \multirow{2}{*}{ CC } & GO:0016021 integral to membrane & 20 & $3.34 E-07$ \\
\hline & & GO:0031224 intrinsic to membrane & 20 & $6.53 E-07$ \\
\hline & MF & GO:0004984 olfactory receptor activity & 20 & $4.36 E-21$ \\
\hline & Pathway & mmu04740:olfactory transduction & 16 & $2.06 E-12$ \\
\hline \multirow{12}{*}{ Module 2} & \multirow{3}{*}{ BP } & GO:0007049 cell cycle & 10 & $4.37 E-08$ \\
\hline & & GO:0006259 DNA metabolic process & 9 & $4.45 E-08$ \\
\hline & & GO:0006260 DNA replication & 6 & $1.68 E-06$ \\
\hline & \multirow{3}{*}{ CC } & GO:0005694 chromosome & 6 & $3.93 E-05$ \\
\hline & & GO:0044427 chromosomal part & 5 & $3.36 E-04$ \\
\hline & & GO:0043228 non-membrane-bounded organelle & 8 & 0.002514 \\
\hline & \multirow{3}{*}{ MF } & GO:0003677 DNA binding & 9 & $7.05 E-05$ \\
\hline & & GO:0005524 ATP binding & 7 & 0.001417 \\
\hline & & GO:0032559 adenyl ribonucleotide binding & 7 & 0.001508 \\
\hline & \multirow{3}{*}{ Pathway } & mmu03030:DNA replication & 3 & 0.001267 \\
\hline & & mmu03420:nucleotide excision repair & 3 & 0.00191 \\
\hline & & mmu04110:cell cycle & 3 & 0.016042 \\
\hline
\end{tabular}

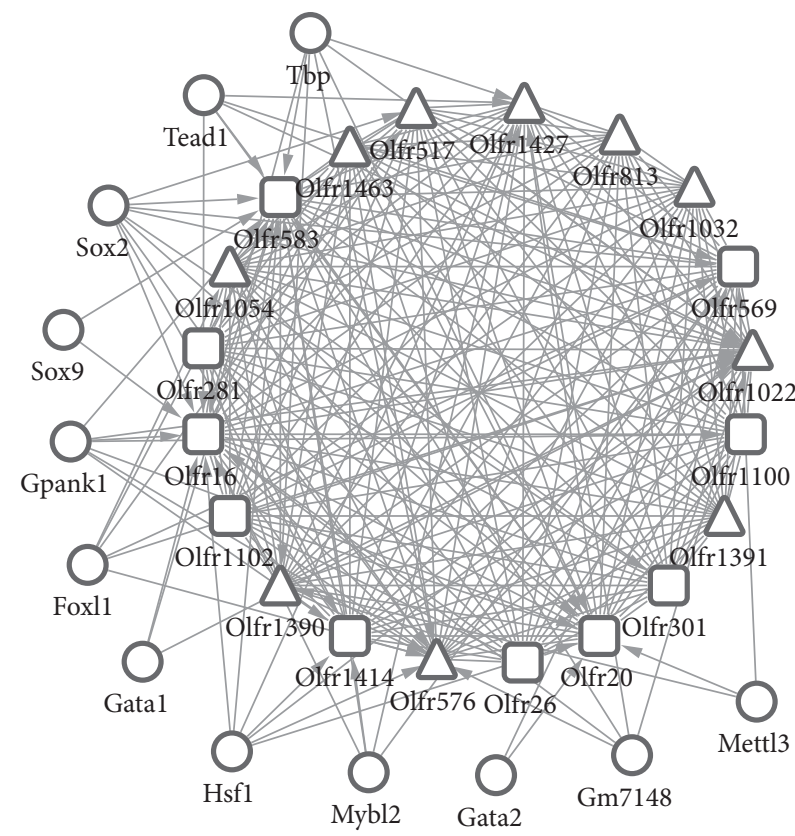

(a)

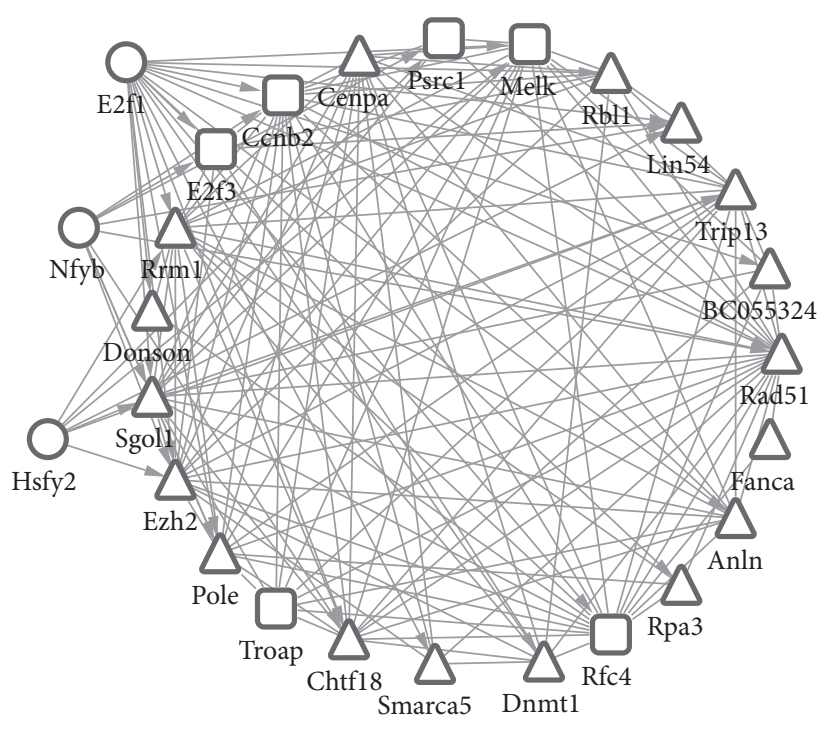

(b)

Figure 3: The significant modules and their related TFs. (a) Module 1; (b) module 2; TFs: transcriptional factors. The trigon represents upregulated genes and the square represents downregulated genes. The roundness means the transcriptional factors.

the barrier function of epithelial monolayers [18]. The concentration dependent experiment has shown that IFN in high concentration diminishes the monolayer resistance by increasing the tight junction permeability [18]. IFN- $\gamma$ has been found to affect the actin distribution and tight junction permeability is affected by cytoskeletal rearrangements
[19]. IFN- $\gamma$ may decrease the epithelial barrier function by rearranging cytoskeletal actin. It is in line with our finding that the actin cytoskeleton was activated by upregulated genes in PHY906 treated colon tumors. Our work also found that IFN- $\gamma$ production was decreased by involvement with downregulated genes, which could illustrate that PHY906 
treatment protected the epithelial barrier against tumor cell invasion by modulating IFN- $\gamma$ level.

In this paper, response to steroid hormone stimulus was significantly enriched by upregulated genes. Steroid hormone is suggested to be a major regulator in cell apoptosis [20]. Previous data has indicated that antiprogestins initiate terminal differentiation and contribute to cell death in breast cancer cells [21]. A line of evidence also shows that apoptosis plays a critical role in tumor cells response to the therapy of cancer [22]. The apoptotic response is determined by the response of tumor cells to the susceptibility of antitumor therapies such as radiation and chemotherapy [23]. The heterogeneity of antitumor drug susceptibility is well correlated with genetic and hormonal regulation and the apoptotic response is initiated by various hormone stimuli [20]. Our work suggested that steroid hormone stimulus was upregulated in PHY906 treated tumor tissues and we speculated that PHY906 induced cancer cell death by activating the response to steroid hormone stimulus.

The original paper suggested that PHY906 directly modulated the cancer cell survival in cell cycle and apoptosis in tumor cells, which is similar to our findings. PPI network analysis also showed that the genes in module 2 were closely related to cell cycle and genes in module 1 were significantly enriched in sensory perception of chemical stimulus. It is reported that PHY906 reduces chemotherapy-induced gastrointestinal toxicity in colon cancer treatment [4]. The cell cycle and sensory perception of chemical stimulus were altered in tumor tissues induced by PHY906, which further indicated that the PHY906 could mediate cancer cell death by modulating cell cycle and sensory perception of chemical stimulus.

Moreover, Ezh2 was found to be the key node in PPI network according to both degree and betweenness. Ezh2 was also clustered in module 2. A line of evidence showed that Ezh2 played key role in stimulating cell growth and proliferation in colon cancer [24]. The expression of Ezh2 is reported to be closely related to treatment response and prognosis of colon cancer patients [25]. Ezh2 has been proposed to be therapeutic target for colon cancer intervention [24]. Besides, Ezh2 is predicted to be regulated by the 3 TFs in module 2, such as E2f1, Hsfy2, and Nfyb. E2f1 is a proliferation promoting TF and has been found to be accumulated in colon cancer [26]. HSF family members have been found to play key roles in proteostasis of cancers [27], while the role of Nfyb in colon cancer has been reported rarely. Thus, the TFs of E2f1, Hsfy2, and Nfyb may play key roles in colon cancer and the functions should be further studied.

Despite the significant findings in this paper, the microarray data analyzed were derived from murine colon tumor tissues. It is unknown whether the similar findings can be produced over human colon cancer tissues. It is a limitation in the present study and further analyses based on human samples are warranted in the near future.

In conclusion, PHY906 showed characteristic in controlling cell apoptosis by intervening interferon-gamma production and response to steroid hormone stimulus. The significant node of Ezh2 and its TFs such as E2f1, Hsfy2, and Nfyb may show the potential therapeutic effect on colon cancer. Our study may provide insights to understand the role of PHY906 in treating colon cancer and provide valuable information for novel anticancer agents development in the future. However, further studies are urgently needed.

\section{Conflicts of Interest}

The authors declare that they have no conflicts of interest.

\section{References}

[1] J. Ferlay, I. Soerjomataram, R. Dikshit et al., "Cancer incidence and mortality worldwide: sources, methods and major patterns in GLOBOCAN 2012," International Journal of Cancer, vol. 136, no. 5, pp. E359-E386, 2015.

[2] L. Ricci-Vitiani, D. G. Lombardi, E. Pilozzi et al., "Identification and expansion of human colon-cancer-initiating cells," Nature, vol. 445, no. 7123, pp. 111-115, 2007.

[3] K. Y. Tan, C. B. Liu, A. H. Chen, Y. J. Ding, H. Y. Jin, and F. SeowChoen, "The role of traditional Chinese medicine in colorectal cancer treatment," Techniques in Coloproctology, vol. 12, no. 1, pp. 1-6, 2008.

[4] W. Lam, S. Bussom, F. Guan et al., "The four-herb Chinese medicine PHY906 reduces chemotherapy-induced gastrointestinal toxicity," Science Translational Medicine, vol. 2, no. 45, pp. 45-59, 2010.

[5] M. Ye, S.-H. Liu, Z. Jiang, Y. Lee, R. Tilton, and Y.-C. Cheng, "Liquid chromatography/mass spectrometry analysis of PHY906, a Chinese medicine formulation for cancer therapy," Rapid Communications in Mass Spectrometry, vol. 21, no. 22, pp. 35933607, 2007.

[6] M. P. Farrell and S. Kummar, "Phase I/IIA randomized study of PHY906, a novel herbal agent, as a modulator of chemotherapy in patients with advanced colorectal cancer," Clinical Colorectal Cancer, vol. 2, no. 4, pp. 253-256, 2003.

[7] E. Wang, S. Bussom, J. Chen et al., "Interaction of a traditional Chinese Medicine (PHY906) and CPT-11 on the inflammatory process in the tumor microenvironment," BMC Medical Genomics, vol. 4, article 38, 2011.

[8] G. K. Smyth, Limma: Linear Models for Microarray Data. Bioinformatics and Computational Biology Solutions Using $R$ and Bioconductor, Springer, 2005.

[9] D. W. Huang, B. T. Sherman, and R. A. Lempicki, "Systematic and integrative analysis of large gene lists using DAVID bioinformatics resources," Nature Protocols, vol. 4, no. 1, pp. 44-57, 2009.

[10] D. Szklarczyk, A. Franceschini, S. Wyder et al., "STRING v10: protein-protein interaction networks, integrated over the tree of life," Nucleic Acids Research, vol. 43, pp. D447-D452, 2015.

[11] X. Tang, J. Wang, J. Zhong, and Y. Pan, "Predicting essential proteins based on weighted degree centrality," IEEE/ACM Transactions on Computational Biology and Bioinformatics, vol. 11, no. 2, pp. 407-418, 2014.

[12] K. I. Goh, E. Oh, B. Kahng, and D. Kim, "Betweenness centrality correlation in social networks," Physical Review E, vol. 67, Article ID 017101, 2003.

[13] E. Estrada and J. A. Rodríguez-Velázquez, "Subgraph centrality in complex networks," Physical Review E, vol. 71, no. 5, 2005.

[14] Y. Tang, M. Li, J. Wang, Y. Pan, and F.-X. Wu, "CytoNCA: a cytoscape plugin for centrality analysis and evaluation of 
protein interaction networks," BioSystems, vol. 127, pp. 67-72, 2015.

[15] T. Nepusz, H. Yu, and A. Paccanaro, "Detecting overlapping protein complexes in protein-protein interaction networks," Nature Methods, vol. 9, no. 5, pp. 471-472, 2012.

[16] R. Janky, A. Verfaillie, H. Imrichová et al., "iRegulon: from a gene list to a gene regulatory network using large motif and track collections," PLoS Computational Biology, vol. 10, no. 7, Article ID e1003731, 2014.

[17] F. Qi, A. Li, Y. Inagaki et al., "Chinese herbal medicines as adjuvant treatment during chemo-or radio-therapy for cancer," Bioscience Trends, vol. 4, pp. 297-307, 2010.

[18] J. L. Madara and J. Stafford, "Interferon- $\gamma$ directly affects barrier function of cultured intestinal epithelial monolayers," The Journal of Clinical Investigation, vol. 83, no. 2, pp. 724-727, 1989.

[19] M. S. Blum, E. Toninelli, J. M. Anderson et al., "Cytoskeletal rearrangement mediates human microvascular endothelial tight junction modulation by cytokines," American Journal of Physiology-Heart and Circulatory Physiology, vol. 273, no. 1, pp. H286-H294, 1997.

[20] W. Kiess and B. Gallaher, "Hormonal control of programmed cell death/apoptosis," European Journal of Endocrinology, vol. 138, no. 5, pp. 482-491, 1998.

[21] H. Michna, Y. Nishino, G. Neef, W. L. McGuire, and M. R. Schneider, "Progesterone antagonists: tumor-inhibiting potential and mechanism of action," Journal of Steroid Biochemistry and Molecular Biology, vol. 41, no. 3-8, pp. 339-348, 1992.

[22] I. Herr and K.-M. Debatin, "Cellular stress response and apoptosis in cancer therapy," Blood, vol. 98, no. 9, pp. 2603-2614, 2001.

[23] L. Milas, L. Stephens, and R. Meyn, "Relation of apoptosis to cancer therapy," In Vivo, vol. 8, pp. 665-673, 1993.

[24] B. Fussbroich, N. Wagener, S. Macher-Goeppinger et al., "EZH2 depletion blocks the proliferation of colon cancer cells," PLoS ONE, vol. 6, no. 7, Article ID e21651, 2011.

[25] O. Fluge, K. Gravdal, E. Carlsen et al., "Expression of EZH2 and $\mathrm{Ki}-67$ in colorectal cancer and associations with treatment response and prognosis," British Journal of Cancer, vol. 101, no. 8, pp. 1282-1289, 2009.

[26] G. Murillo, G. I. Salti, J. W. Kosmeder II, J. M. Pezzuto, and R. G. Mehta, "Deguelin inhibits the growth of colon cancer cells through the induction of apoptosis and cell cycle arrest," European Journal of Cancer, vol. 38, no. 18, pp. 2446-2454, 2002.

[27] C. Dai and S. B. Sampson, "HSF1: guardian of proteostasis in cancer," Trends in Cell Biology, vol. 26, no. 1, pp. 17-28, 2016. 


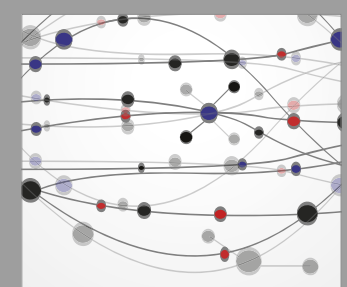

The Scientific World Journal
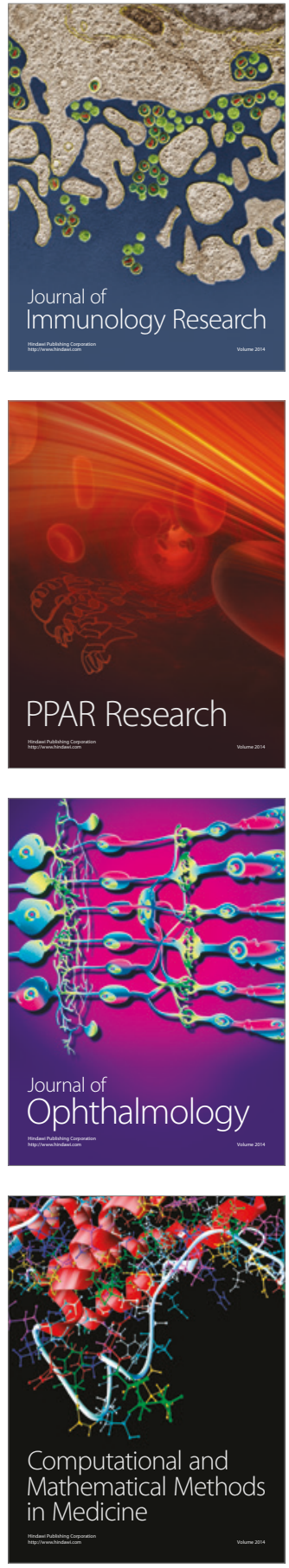

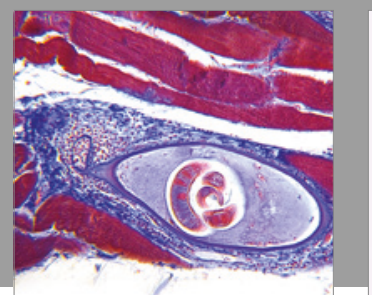

Gastroenterology Research and Practice
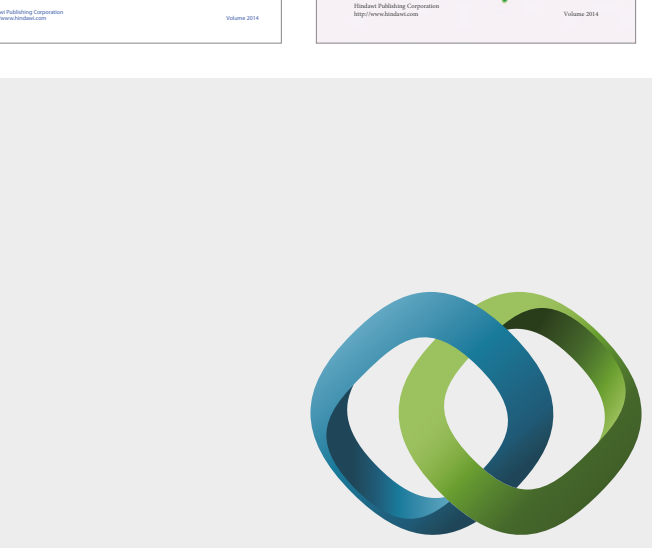

\section{Hindawi}

Submit your manuscripts at

https://www.hindawi.com
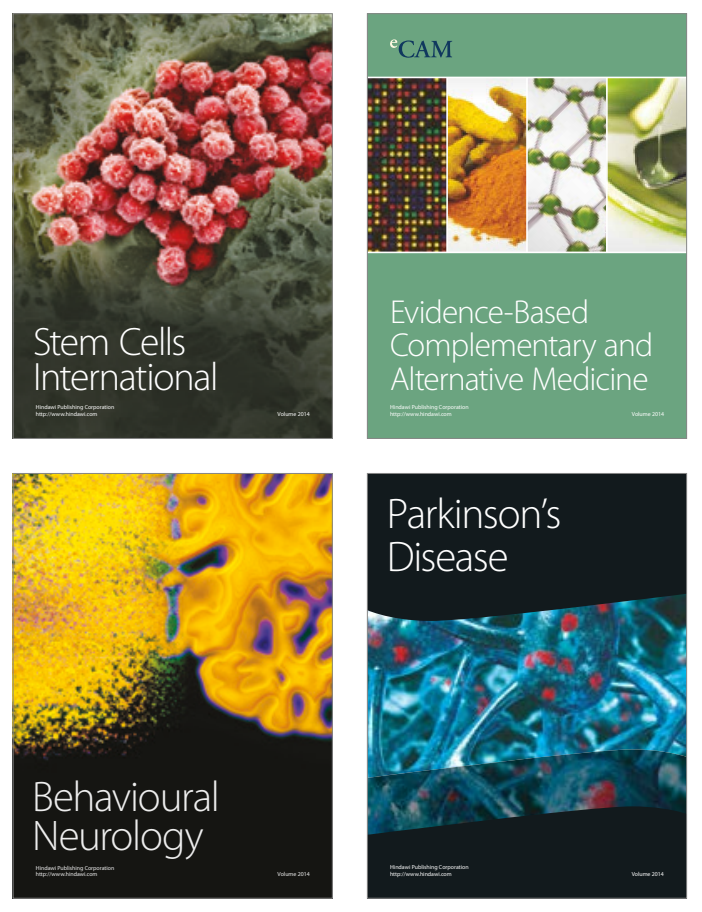
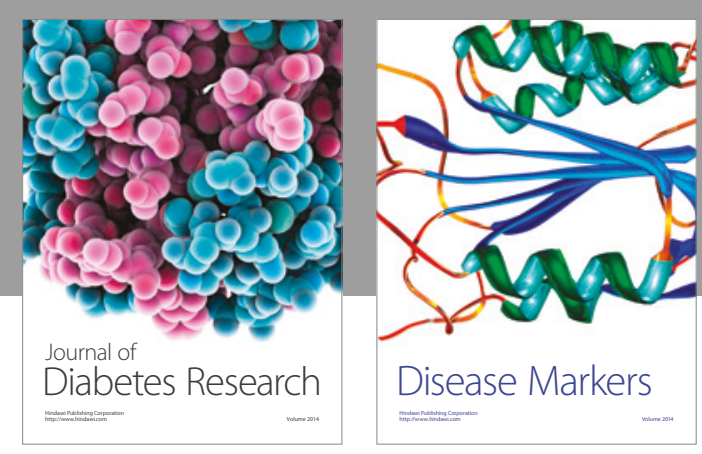

Disease Markers
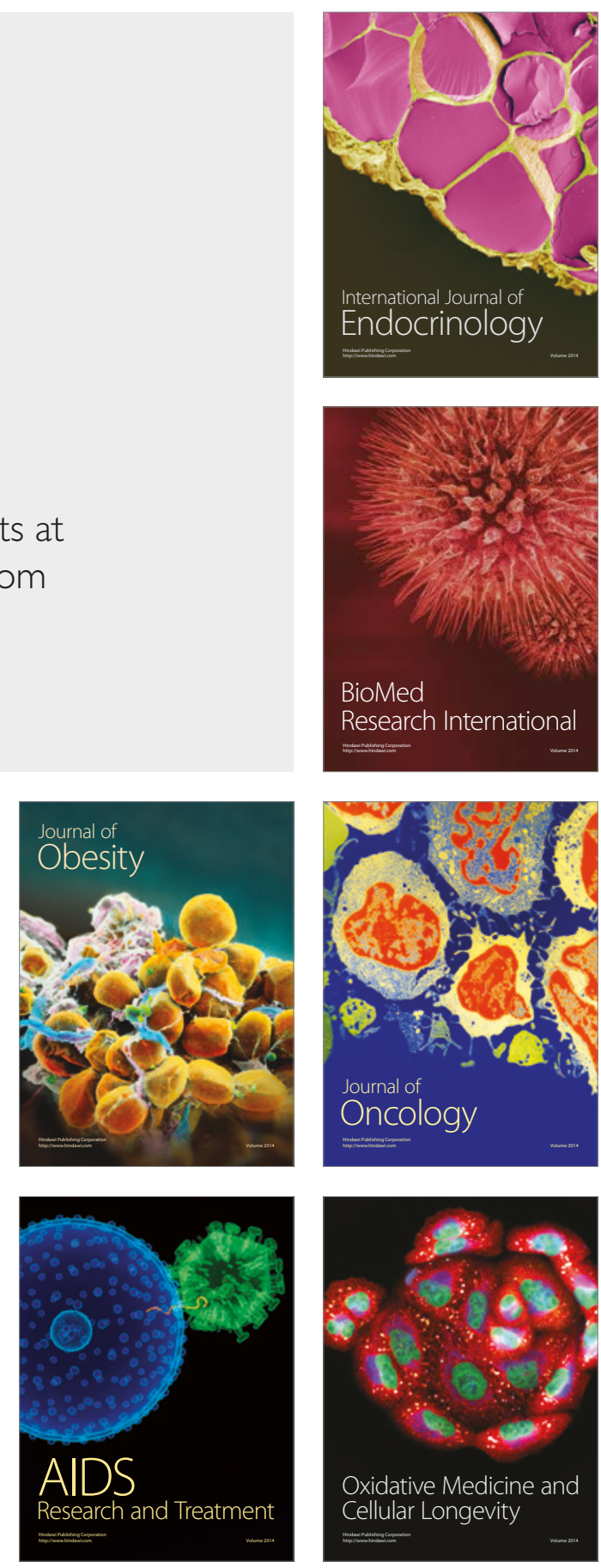